\title{
Konzept zur zielgerichteten Kompetenzentwicklung für Initiativen des Maschinellen Lernens
}

\author{
Thorben Panusch ${ }^{1(\varangle)}$, Jan Büscher ${ }^{1}$, René Wöstmann ${ }^{1}$, und Jochen Deuse ${ }^{1,2}$ \\ ${ }^{1}$ Institut für Produktionssysteme, TU Dortmund, Leonhard-Euler-Straße 5, \\ 44227 Dortmund, Deutschland \\ $\{$ Thorben. Panusch, Jan.Buescher, Rene.Woestmann, \\ Jochen. Deuse\} \&ips. tudortmund. de \\ Jochen. Deuseduts. edu. au \\ ${ }^{2}$ Centre for Advanced Manufacturing, University of Technology Sydney, \\ Sydney, Australia
}

\begin{abstract}
Zusammenfassung. Durch die fortschreitende Digitalisierung und Automatisierung sind Unternehmen einem stetigen Transformationsprozess ausgesetzt. Dadurch entfallen alte Berufsbilder und gleichzeitig entstehen völlig neue Berufe mit veränderlichen und digitalen Kompetenzanforderungen. Um zu verhindern, dass der technologische Wandel mit einem Talentmangel, Massenarbeitslosigkeit und wachsender Ungleichheit einhergeht, müssen Unternehmen eine aktive Rolle bei der Unterstützung ihrer bestehenden Belegschaft durch Weiterbildung und Höherqualifizierung übernehmen. Dies ist besonders wichtig, da es einen positiven Kreislauf zwischen neuen Technologien und Weiterbildungen gibt. Die Einführung neuer Technologien fördert das Unternehmenswachstum, die Schaffung neuer Arbeitsplätze und den Ausbau bestehender Arbeitsplätze. Voraussetzung dafür ist, dass Mitarbeiter durch kontinuierliche Umschulung und Weiterbildung mit zukunftssicheren Fähigkeiten ausgestattet sind, um neue Chancen wahrzunehmen. Hierzu zählen besonders Kompetenzen im Bereich des Maschinellen Lernens (ML), das bisher ungeahnte Potenziale zur Wissensgewinnung eröffnet und so einen entscheidenden Erfolgsfaktor für Unternehmen bilden kann. Der Aufbau von digitalen Kompetenzen stellt jedoch für Unternehmen eine signifikante Herausforderung dar, besonders für kleine und mittlere Unternehmen. Oft fehlen für Weiterbildungen die zeitlichen und finanziellen Freiräume oder das notwendige Know-how. Zudem ist der Weiterbildungsmarkt äußerst unübersichtlich, was die Identifikation geeigneter Weiterbildungen erschwert. Um diese Problematik zu adressieren, wird in diesem Beitrag ein Konzept vorgestellt, das Unternehmen unterstützen soll, geeignete Maßnahmen zum zielgerichteten Aufbau von ML-Kompetenzen abzuleiten. Dies eröffnet Unternehmen einen Überblick über erforderliche Kompetenzen und Rollen, die mit den vorhandenen Kompetenzen im Unternehmen abgeglichen werden können. Ferner
\end{abstract}


werden aktuelle Weiterbildungsmöglichkeiten vorgestellt, auf deren Grundlage ein Schulungskatalog aufgebaut wird. Dieser erlaubt es, die identifizierten Kompetenzlücken mit geeigneten Weiterbildungen zu adressieren.

Schlüsselwörter: Kompetenzentwicklung · Maschinelles Lernen · Digitale Souveränität

\section{Einleitung}

Im letzten Jahrzehnt hat eine Reihe von bahnbrechenden, neu entstandenen Technologien den Beginn der vierten industriellen Revolution eingeläutet (Zahidi et al. 2020). Da sich dadurch die Grenzen zwischen den von Menschen und den von Maschinen sowie Algorithmen ausgeführten Arbeitsaufgaben rapide verschieben, sind die globalen Arbeitsmärkte einem tiefgreifenden Wandel unterworfen. Im Zuge dieser Entwicklung versuchen Unternehmen, neue und aufkommende Technologien zu nutzen, um eine höhere Effizienz in der Produktion zu erreichen, in neue Märkte zu expandieren und mit neuen Produkten um eine globale Kundenbasis zu konkurrieren (Leopold et al. 2018).

Im Mittelpunkt steht dabei die zunehmende Digitalisierung und Verbreitung von Informations- und Kommunikationstechnologien (IKT), wodurch der Aufbau dynamischer Datenspeicher bisher unbekannter Größe und Güte ermöglicht wird (Eickelmann et al. 2015). Die Auswertung und effiziente Nutzung des in den gespeicherten Datenmengen implizit vorhandenen Wissens zur Entscheidungsunterstützung wird in produzierenden Unternehmen immer wichtiger. Eine Möglichkeit dazu bieten die Methoden des Maschinellen Lernens (ML). Diese ermöglichen, nicht triviale und bisher unbekannte Strukturen und Zusammenhänge in großen Datenmengen zu entdecken (Deuse et al. 2014; Fayyad et al. 1996). Die so gewonnenen Erkenntnisse bilden in Kombination mit dem praktischen Erfahrungswissen der Mitarbeiter zukünftig einen wesentlichen Erfolgsfaktor für Unternehmen (Deuse et al. 2014).

Durch die industrielle Transformation und die Einbringung neuer Technologien verändern sich allerdings auch die Kompetenzanforderungen an die Mitarbeiter der Unternehmen (Boockmann et al. 2021; Leopold et al. 2018). Dieser Trend hat sich durch die Coronakrise nochmals beschleunigt. Dabei prognostizierte das Weltwirtschaftsforum 2020, dass bis 2025 weltweit 85 Mio. Arbeitsplätze durch die Automatisierung entfallen, während 97 Mio. völlig neue Berufe an der Schnittstelle Mensch, Maschine und Algorithmus entstehen. Doch auch bei den Mitarbeitern, die in ihren Funktionen verbleiben, werden sich die Kernqualifikationen um $40 \%$ ändern. Insgesamt werden so $50 \%$ aller Angestellten eine Weiterbildung benötigen (Zahidi et al. 2020). Laut einer Studie mit 3000 befragten Fach- und Führungskräften sehen $59 \%$ jetzt schon einen Mangel an Kompetenzen mit Hinblick auf die digitale Arbeitswelt. $64 \%$ unter den befragten Führungskräften und Personalern erwarten sogar, dass 
die Kompetenzlücken noch größer werden. Dabei fehlen Kompetenzen besonders im Bereich der IT und Datenanalyse (Dettmers et al. 2021). Dieser Mangel ist in kleinen und mittleren Unternehmen (KMU) noch gravierender als in großen Unternehmen (Czernich et al. 2019).

Um beschäftigungsfähig zu bleiben und berufliche Entwicklungschancen nutzen zu können, ist es daher für Mitarbeiter unerlässlich, durch regelmäßige Weiterbildungen kontinuierlich neues Wissen und neue Kompetenzen zu erwerben und sich so an die neuen beruflichen Anforderungen anzupassen (Boockmann et al. 2021). Zusätzlich sollten Regierungen ein günstiges Umfeld schaffen, um diese Bemühungen zu unterstützen. Gleichzeitig müssen auch Unternehmen durch Umschulungen und Höherqualifizierungen eine aktive Rolle bei der Unterstützung ihrer bestehenden Belegschaft übernehmen. Damit Unternehmen in einer digitalisierten Industrie differenziert, wettbewerbsfähig und digital souverän bleiben, müssen sie in ihr Humankapital investieren, das sie als Vermögenswert und nicht als Belastung betrachten sollten. Nur durch proaktive Ansätze können Unternehmen und Mitarbeiter das wirtschaftliche Potenzial der vierten industriellen Revolution ausschöpfen (Leopold et al. 2018).

Für Unternehmen und im Besonderen für KMU stellen der Aufbau von digitalen Kompetenzen, wie etwa im Bereich des ML, und die Implementierung von Weiterbildungsmaßnahmen große Herausforderungen dar. Voraussetzung, um diese zu meistern, ist das Vorhandensein einer digitalen Strategie. Diese legt die allgemeinen Ziele der im Unternehmen zu entwickelnden oder einzusetzenden digitalen Anwendungen fest. Dazu werden genaue Kenntnisse über die Potenziale dieser Anwendungen benötigt. Die digitale Strategie ist vom digitalen Reifegrad des Unternehmens abhängig, wobei vorab geprüft werden muss, in welchen Bereichen es überhaupt technisch und operativ möglich ist, digitale Methoden wie des ML anzuwenden. In KMU ist eine solche digitale Strategie häufig jedoch nicht vorhanden (Plattform Lernende Systeme 2021). Laut einer repräsentativen Umfrage haben erst $40 \%$ überhaupt die erforderlichen Digitalkompetenzen identifiziert. Überdies bilden nur $20 \%$ der Unternehmen ihre Mitarbeiter unterhalb des höheren Managements in digitalen Kompetenzen weiter. Hierfür sind mehrere Gründe verantwortlich. So mangelt es oft an dem nötigen Know-how über digitale Innovationspotenziale, wodurch Innovationsprozesse und digitale Strategien nicht initiiert werden können. Des Weiteren fehlen die zeitlichen Freiräume, um sich mit Weiterbildungen auseinanderzusetzen und diese durchzuführen. Außerdem stehen dafür nicht immer genügend finanzielle Mittel zur Verfügung, was durch die Coronakrise noch verschärft wurde. Vor allem für KMU sind das häufige Hindernisse. Ein weiteres Problem ist die Intransparenz des Weiterbildungsmarktes, die es Unternehmen erschwert, ein passendes Angebot zu identifizieren. Es gibt rund 18.000 Anbieter, darunter staatliche Bildungsträger, Privatakademien, Berufsverbände, Gewerkschaften und Arbeitgeberorganisationen (Bertelsmann Stiftung 2018; Morik et al. 2010; Nöhring 2021; Obmann 2021).

Generell besteht zwischen Industrie und Forschung ein Konsens über die Bedeutung eines gezielten Aufbaus digitaler Kompetenzen, insbesondere im Bereich 
des ML (acatech 2016). Auch die Regierung unterstützt das Bestreben und hat dafür 2019 das Qualifizierungschancengesetz erlassen (Obmann 2021; Gillmann 2021). Allerdings benötigen Unternehmen und im Speziellen KMU aufgrund der beschriebenen Probleme Unterstützung bei der Weiterbildung von Mitarbeitern in digitalen Themen wie ML. Aus diesem Grund wurde ein Konzept entwickelt, das Unternehmen erlaubt, zielgerichtet Maßnahmen zum Aufbau von Kompetenzen abzuleiten, die für die Umsetzung von ML-Anwendungen notwendig sind.

Hierfür werden in Abschn. 2 die einzelnen Rollen und Kompetenzen ausgeprägt, die für das interdisziplinäre Arbeiten bei der Umsetzung von ML-Projekten erforderlich sind. Dies ermöglicht Unternehmen, einen Überblick über die erforderlichen Kompetenzen einer bestimmten Rolle in Form eines Zielprofils zu erlangen. Um etwaige Kompetenzlücken mithilfe eines Soll-Ist-Vergleichs zu identifizieren, müssen die vorhandenen Kompetenzen der entsprechenden Mitarbeiter zunächst erfasst werden. Zu diesem Zweck werden in Abschn. 3 die wissenschaftlichen Methoden zur Kompetenzerfassung vorgestellt und diskutiert. Darauf aufbauend wird die optimale Methode für den vorliegenden Anwendungsfall ausgewählt. In Abschn. 4 wird ein allgemeiner Überblick über das aktuelle Angebot an Weiterbildungen gegeben. Dies soll zum einen Klarheit über den Weiterbildungsmarkt bringen. Zum anderen werden die verschiedene Angebote mit ihren unterschiedlichen Charakteristiken für den Aufbau eines Schulungskatalogs verwendet. Dieser wird im Rahmen des Konzepts entwickelt, um mithilfe einer zielgerichteten Ableitung von Weiterbildungen identifizierte Kompetenzlücken zu adressieren. In Abschn. 5 wird dann das erarbeitete Konzept vorgestellt, das die Vorarbeiten aus den Abschn. 2, 3 und 4 zusammenführt. Der Beitrag schließt in Abschn. 6 mit einer kurzen Zusammenfassung und einem Ausblick auf die weiteren Schritte ab.

\section{Rollen und Zielkompetenzen interdisziplinärer ML-Teams}

Die Differenzierungsmöglichkeiten von Berufsbilden und erforderlichen Kompetenzprofilen für die zukünftige Arbeitswelt in produzierenden Unternehmen und insbesondere im Maschinen- und Anlagenbau sind vielfältig und Gegenstand diverser Forschungsarbeiten. Reckelkamm und Deuse (2021) leiten basierend auf einer Literaturrecherche die Kategorien der Fach-, Sozial-, Methoden- und Selbstkompetenz als wesentliche Handlungsfelder ab. Detailliertere Analysen zu erforderlichen ML-Kompetenzen in der produzierenden Industrie bieten Schulte et al. (2020), Zschech et al. (2018) und Bauer et al. (2018).

Da Produkte, Prozesse und Anlagen, und damit verbunden ebenfalls Probleme, Lösungsmuster und Services, zunehmend komplexer werden, gewinnen interdisziplinäre Teamzusammenstellungen an Bedeutung (Saltz und Grady 2017). Obwohl die identifizierten Kompetenzen für einen allgemeinen Überblick nützlich sind, müssen jedoch Rollen und Akteure gleichermaßen spezifiziert werden. In der 
Literatur finden sich sowohl theoretische Modelle für die generelle Zusammensetzung von ML-Teams, beispielsweise Saltz und Grady (2017), als auch praxisorientierte Leitfäden, beispielsweise RapidMiner (2020). Diese adressieren jedoch die Domäne der Produktion nur unzureichend oder verlieren sich in Details.

Um diese Lücke zu schließen, wurde basierend auf den Arbeiten von Schulte et al. (2020), Stark et al. (2019) und Reckelkamm und Deuse (2021) ein Rollenmodell zur Bildung interdisziplinärer Kompetenzprofile erarbeitet. Es besteht aus den Rollen IT, Domänenexperte, Data Scientist und Management, deren jeweilige Aufgaben durch einen ausgewählten Citizen Data Scientist in einer zentralen Orchestrierungsrolle integriert werden. Eine detaillierte Vorstellung der Aufgabenprofile erfolgt in Deuse et al. (2021). Die Grundlage sowohl für die Abgrenzung der Rollen als auch für die Ausgestaltung der Kompetenzprofile bildeten iterative Workshops mit Anwendern und Führungskräften aus der produzierenden Industrie, Maschinen- und Anlagenbauern, Data Scientists, IT-Unternehmen sowie Forschungseinrichtungen. Die Ausprägungen der Kompetenzprofile wurden differenziert in keine Kompetenz (0) und grundlegende Informationen (1), die durch Anwendung und Vernetzung zu Wissen (2) werden. Dieses wird durch Bereitschaft und praktische Erfahrung zur Kompetenz (3) erweitert. Die höchste Stufe (4) adressiert die Fähigkeit zur Synthese und Bewertung unterschiedlicher Ansätze und Disziplinen. Die Abstufungen orientieren sich somit an der Wissenstreppe nach North et al. (2016) sowie dem Stufen-Taxonomie-Modell nach Bloom und Engelhart (1976). In Abb. 1 werden die Rollen sowie deren spezifische Kompetenzprofile detailliert vorgestellt. Die teilweise ungeraden Werte der Kompetenzstufen im folgenden Abschnitt resultieren aus dem Prozess der Einzelbefragung von Industrie- und Forschungsexperten und der anschließenden Kombination der Bewertungen, um ein valideres Gesamtbild zu erhalten.

Die ausgeprägten Rollen vereinen unterschiedliche charakteristische Kompetenzprofile, die auf ihre individuellen Anforderungen ausgerichtet sind. Während die Kompetenzanforderungen des Data Scientist insbesondere Methoden und Verfahren des ML und der Statistik adressieren, bündeln IT-Abteilungen Kompetenzen im Datenmanagement sowie in der Informations- und Kommunikationstechnik. Daneben bringen Domänenexperten ihr spezifisches Domänenwissen in die Problemspezifizierung und die Bewertung von Lösungen ein. Das Management initiiert und evaluiert primär Projekte, nimmt jedoch keine aktive Rolle in der Bearbeitung ein. Es bedarf daher keiner ausgeprägten Fachkompetenzen, verfügt aber über höhere Sozial-, Selbst- und besonders Methodenkompetenzen. Der Orchestrierer, der von einem ausgewählten Citizen Data Scientist verkörpert wird, ist der einzige Akteur, der Grundlagen aller Disziplinen und somit Fachkompetenzen vereint. Da er zusätzlich aber auch im operativen Projektmanagement in der Verantwortung steht, hat er für die erfolgreiche Durchführung von Data-Science-Initiativen hohe Methoden-, Sozial- und Selbstkompetenzen aufzuweisen. 


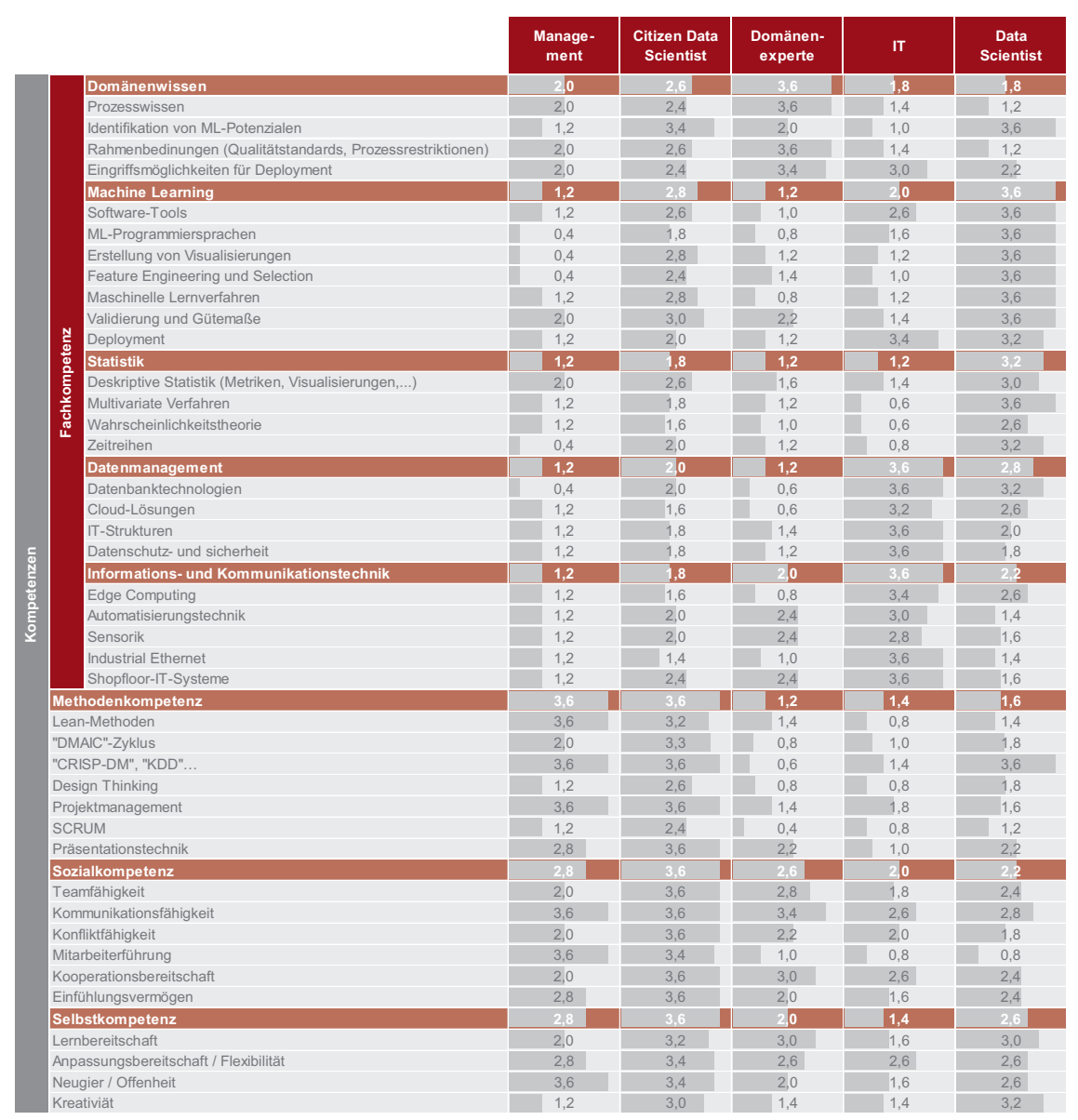

Abb. 1. Kompetenzprofile interdisziplinärer ML-Teams in der Produktion

\section{Erfassung vorhandener Kompetenzen}

Um in Zeiten der digitalen Transformation die Hoheit über Daten und Wissen zu behalten, müssen schlagkräftige Teams zur Entwicklung von neuartigen Produkten und Services in KMU verfügbar sein. Diese Tatsache steht gleichzeitig einem Kompetenz- und Fachkräftemangel in den beschriebenen Bereichen gegenüber, sodass Weiterbildungsmaßnahmen systematisch durchgeführt werden müssen. Grundlegend für den Aufbau und die Gewährleistung von erforderlichen Kompetenzen in ML-Teams ist daher zunächst, dass die vorhandenen Kompetenzausprägungen der entsprechenden Teammitglieder systematisch erfasst werden können. Im Kontext der Human- und Sozialwissenschaften unterscheiden Döring und Bortz (2016) verschiedene Datenerhebungsmethoden. Dazu zählen die Beobachtung, der psychologische Test, die mündliche Befragung (Interview), die schriftliche Befragung 
(Fragebogen), die physiologische Messung und die Dokumentenanalyse. Für die Kompetenzerfassung sind nur die ersten vier Methoden geeignet. Bei physiologischen Messungen werden Merkmale körperlicher Prozesse in den menschlichen Organen gemessen. Bei der Dokumentenanalyse werden historische Dokumente ausgewertet, was eher durch einen qualitativen Charakter geprägt ist und in der Regel keine direkte Quantifizierung von Merkmalen erlaubt. Beide Methoden sind für eine präzise Erfassung von Kompetenzen nicht geeignet. Als Voraussetzung für alle Methoden ist im Rahmen der Operationalisierung ein standardisiertes Erhebungsinstrument, wie beispielsweise ein Beobachtungsplan, Interviewleitfaden, Fragebogen oder Kategoriensystem, zu erstellen. Dazu wird zur Durchführung der Datenerhebung teilweise geschultes Personal benötigt (Döring und Bortz 2016). Insbesondere für KMU besteht hierbei der Bedarf, eine Erhebungsmethode zu verwenden, die einerseits möglichst wenig Aufwand erfordert und andererseits eine hinreichende Validität hinsichtlich der spezifizierten persönlichen und sachlich-theoretischen Ausprägungen bietet.

Die Datenerhebung in Form der wissenschaftlichen Beobachtungen zeichnet sich durch eine ,direkte (...) Beobachtung allein auf Basis menschlicher Sinnesorgane" (Döring und Bortz 2016) aus. Sie ist also auf einen wissenschaftlichen Beobachter angewiesen und ist damit vergleichsweise zeit- und kostenaufwendig. Zudem sind viele subjektive Erlebensphänomene durch eine Fremdbeobachtung nicht erfassbar und müssen daher erfragt werden. Die Beobachtung stellt für die Kompetenzerfassung von Mitarbeitern bei KMU keine geeignete Methode dar. Diese Unternehmen können normalerweise nicht auf einen wissenschaftlichen Beobachter zurückgreifen. Ferner sind der zeitliche und monetäre Aufwand gegenüber anderen Methoden der Datenerhebung, wie der Befragung, zu hoch. Auch die Einschränkungen durch die Fremdwahrnehmung stellen eine wesentliche Limitierung bezüglich der Validität dar (Döring und Bortz 2016).

Psychologische Tests eignen sich besonders, um die exakte Ausprägung eines bestimmten Merkmals einer Person zu erheben, die sich durch Beobachtungen oder Selbstauskünfte nur schwer erfassen lassen. Sie sind durch eine Reihe von empirisch geprüften und vollstandardisierten Testaufgaben gekennzeichnet, die von den Testpersonen zu bearbeiten sind, zuweilen unter Zeitdruck. Psychologische Tests werden daher häufig im Bereich der Diagnostik zur psychologischen Beurteilung eingesetzt. Grundsätzlich werden sie in Persönlichkeits- und Leistungstests unterschieden. Bei den Persönlichkeitstests werden persönliche Merkmale durch eine Selbstauskunft ermittelt. Sie weisen zwar eine hohe Ähnlichkeit zu vollstandardisierten Fragebögen auf, unterliegen jedoch einer deutlich gründlicheren Überprüfung der Testgütekriterien. Leistungstests erfordern das Lösen von Testaufgaben. Dabei werden Normwerte aufgestellt, die aus Testergebnissen repräsentativer Bevölkerungsgruppen abgeleitet werden. Diese Normierung ist Grundvoraussetzung für die Beurteilung einer Einzelperson. Ein wesentlicher Nachteil von psychologischen Tests ist der erhebliche Aufwand. Dies gilt zum einen für die Erstellung und systematische Auswertung der individuellen Tests, die infolge fehlender Expertise nicht durch die Unternehmen selbst erfolgen kann. Zum anderen gilt dies auch für die Testpersonen aufgrund der umfangreichen und hohen Anzahl an Testaufgaben. Außerdem müssen Leistungstests unter Aufsicht abgehalten werden. Darüber hinaus sind psychologische Tests relativ kostenintensiv, die oft für jede Testperson Kosten verursachen. Für den 
vorliegenden Anwendungsfall sind psychologische Test somit trotz hoher Validität als $\mathrm{zu}$ aufwendig und kostenintensiv einzuordnen und deshalb nicht geeignet. (Döring und Bortz 2016)

Strukturierte mündliche und schriftliche Befragungen sind die verbreitetsten Formen der Datenerhebung in den empirischen Sozialwissenschaften. Die mündliche Befragung wird in Form eines Interviews und die schriftliche Befragung mithilfe eines Fragebogens durchgeführt. Als grundlegender Unterschied zwischen beiden Methoden wird bei der mündlichen Befragung eine weitere Person als geschulter Fragesteller benötigt. Daraus resultiert ein erheblich höherer Zeit- und Kostenaufwand. Die mündliche Befragung eignet sich eher bei einer größeren Anzahl an Fragen oder komplizierten Fragestellungen. So können Befragungspersonen mündlich in kürzerer Zeit mehr Informationen angeben sowie komplexe Zusammenhänge ausführlicher schildern. Die Fragebogenmethode ist dagegen durch Selbstadministration wesentlich effizienter. Damit können in kurzer Zeit die Antworten von vielen Befragungspersonen zu vielen Merkmalen gesammelt werden. Allerdings müssen sich Fragebögen im Unterschied zum Interview auf wenige und klar umschriebene Befragungsinhalte konzentrieren. Ferner fehlt die Möglichkeit der individuellen Reaktion, sodass Fragebögen weniger transparent sind als Interviews (Döring und Bortz 2016).

Zusammenfassend stellt die strukturierte Befragung aufgrund der Vergleichbarkeit, des relativ geringen zeitlichen und finanziellen Aufwands sowie der relativ hohen Validität die geeignetste Methode zur Kompetenzerfassung im Rahmen der Konzepterstellung dar. Die Grundlage für die Kompetenzerfassung bilden die in Abb. 1 ausgeprägten Kompetenzen. Gemäß Abschn. 2 sind die einzelnen definierten Rollen mit einem spezifischen Kompetenzprofil verbunden. Um eine jeweilige Rolle hinreichend ausfüllen zu können, müssen demnach die festgelegten Niveaus in den einzelnen Kompetenzen mindestens erreicht werden. Das Kompetenzprofil einer Rolle dient somit als Zielprofil für einen vorgesehenen Mitarbeiter. Folglich muss zur Überprüfung der Tauglichkeit für eine Rolle das vorhandene Niveau des entsprechenden Mitarbeiters in den in Abb. 1 ausgeprägten Kompetenzen nach dem vorgestellten Bewertungsschema von (0) bis (4) erfasst werden. Diese kohärente Kompetenzerfassung ermöglicht es, für ein Zielprofil einen Soll-Ist-Vergleich vorzunehmen. Auf diese Weise können etwaige Defizite in einzelnen Kompetenzen aufgedeckt oder das Erfüllen der Mindestanforderungen festgestellt werden. Da es sich bei der numerischen Bewertung der einzelnen Kompetenzen um ein aufwandsarmes und relativ unkompliziertes Frageschema handelt, empfiehlt es sich, die schriftliche Befragung der mündlichen vorzuziehen und als strukturierten Fragebogen durchzuführen. Die Erfassung der Kompetenzen der jeweiligen Mitarbeiter wird dabei selbstständig in den Unternehmen vorgenommen. Die Bewertung kann durch einen fachlichen und einen disziplinarischen Vorgesetzten in Abstimmung mit dem Mitarbeiter selbst erfolgen. 


\section{Aktuelle Weiterbildungsmöglichkeiten}

In Abschn. 2 wurden die Rollen und die spezifischen Kompetenzen für die Zusammensetzung eines ML-Teams definiert. Dabei sind auch die Kompetenzstufen ausgeprägt, die mindestens erreicht werden müssen, um die jeweilige Rolle ausfüllen zu können. Dies bedeutet aber auch, dass die einzelnen Kompetenzen bei einer etwaigen Diskrepanz gezielt durch passende Weiterbildungen auf das erforderliche Niveau entwickelt werden müssen. Daraus resultiert für KMU der Bedarf nach einer Möglichkeit zur gezielten Entwicklung einzelner Kompetenzen von Mitarbeitern neben der beruflichen Tätigkeit.

Aktuell stehen hierfür diverse Weiterbildungsangebote in verschiedenen Formaten zur Verfügung. Grundsätzlich kann das Angebot in Onlinekurse und Präsenzveranstaltungen differenziert werden, wobei Onlinekurse die Mehrheit der Weiterbildungsmöglichkeiten ausmachen (Zschech et al. 2018).

Eine zentrale Position nehmen dabei Anbieter von Massive Open Online Courses (MOOC) ein, die nach der Einführung 2008 erheblich an Bedeutung gewonnen haben. Das Interesse an diesem Ausbildungsformat ist so groß geworden, dass die New York Times das Jahr 2012 zum ,Jahr des MOOC“ erklärte und Linkedin 2014 gar als das „Jahr des Corporate MOOC“ titulierte (Nielson 2014; Pappano 2012; Siemens 2013). Bei MOOCs handelt es sich um Onlinekurse, die auf einen offenen Zugang über das Internet ausgerichtet sind und eine unbegrenzte Teilnehmerzahl erlauben. Zusätzlich zu traditionellen Kursmaterialien, wie aufgezeichneten Vorlesungen, Lektüren und Übungen, bieten viele MOOCs interaktive Kurse mit Benutzerforen, um die Interaktion zwischen Schülern und Lehrkräften zu unterstützen, sowie unmittelbares Feedback zu Prüfungsfragen und -aufgaben (Kaplan und Haenlein 2016). Ein weiteres entscheidendes Charakteristikum ist die zeitliche Unabhängigkeit, die den Teilnehmern ermöglicht, ihr Lernen je nach Lernzielen, Vorkenntnissen und Fähigkeiten bzw. eigenem Tempo selbst und asynchron zu organisieren. Obwohl manche MOOCs die Konventionen herkömmlicher Kurse mit etwa einem vordefinierten Zeitplan und wöchentlich zu behandelnden Themen teilen mögen, ist ihnen gemein, dass sie nahezu keine Gebühren fordern (McAuley et al. 2010; Pappano 2012). Als Folge können diese neuen Bildungsformate sogar einen direkten Einfluss auf Unternehmen und Organisationen haben. So könnten beispielsweise MOOCs hinreichender Güte problemlos und kosteneffizient in die firmeninterne Weiterbildung oder Corporate University integriert werden. Die Kombination aus Kosteneffizienz und Flexibilität macht dieses digitale Format besonders attraktiv für Unternehmen (Kaplan und Haenlein 2016).

Relevante MOOC-Anbieter sind Udemy, Udacity, Coursera, edX, Pluralsight, FutureLearn, iversity, Alison, LinkedIn Learning, OpenClassrooms, OpenLearning und openHPI. Zudem gibt es auch domänenspezifischere Plattformen, wie DataCamp und Cognitive Class (ehemals Big Data University). Einige Anbieter bieten sogar Kurse in Kooperation mit Universitäten oder Unternehmen an, wobei besonders international renommierte Universitäten sowie große Technologieunternehmen stark vertreten sind (Dodson et al. 2015; Edukatico 2021; Lewin 2013; Pappano 2012). 
Obwohl MOOCs ihre Bekanntheit als Disruptoren der traditionellen Hochschulbildung erlangt haben, drängen MOOC-Anbieter mittlerweile mit speziellen Berufsbildungsangeboten intensiv in den Unternehmenssektor (Dodson et al. 2015). So haben sich einige Anbieter komplett auf die berufliche Weiterbildung ausgerichtet. Zudem haben viele Anbieter inzwischen Weiterbildungsprogramme speziell für Unternehmen erstellt. Unternehmen können hierbei auf Onlinekurse zugreifen und diese nach ihren Bedürfnissen kostenpflichtig auslegen (Castellano 2014). Es werden auch spezielle Angebote für den Unternehmenssektor entwickelt. So wird Unternehmen durch die Nutzung von MOOCs ermöglicht, Kosten für die Mitarbeiterschulung zu senken, Talentpipelines aufzubauen und Jobkandidaten mit nachweisbaren Fähigkeiten zu identifizieren sowie näher an Interessenten und Kunden heranzukommen (Dodson et al. 2015).

Zusätzlich zu dem Angebot der MOOC-Plattformen bieten mittlerweile auch viele Universitäten ihre eigenen Kurse und Vorlesungsreihen als Onlinekurse an. Darunter fallen auch vor allem Eliteuniversitäten, wie das MIT, die Stanford und Harvard University, die dafür neben dem Angebot auf den bekannten MOOC-Plattformen teilweise eigene Portale betreiben oder auf alternative Plattformen, wie YouTube, zurückgreifen. Darüber hinaus stellen große (Tech-)Unternehmen, wie Amazon Web Services, Microsoft, Google, SAP, IBM, SAS und Cloudera, aber auch speziell auf ML ausgerichtete Unternehmen, wie RapidMiner, eigene und auf ihre individuelle Software zugeschnittene Schulungsreihen zur Verfügung.

Es werden weiterhin auch viele traditionelle berufliche Weiterbildungen als Zertifikatskurse angeboten. Dabei gibt es zum einen In-House-Schulungen, die direkt am Arbeitsplatz durchgeführt werden. Zum anderen existieren Weiterbildungen in Form von Kursen, Seminaren und Workshops. Diese werden in der Regel als Präsenzveranstaltungen abgehalten, einige sind aber inzwischen auch als E-Learning-Angebote verfügbar. Zu den Anbietern zählen Hochschulen, die abseits der klassischen Studiengänge zertifizierte Weiterbildungen in Form des weiterbildenden Studiums zur wissenschaftlichen oder künstlerischen Vertiefung und Ergänzung berufspraktischer Erfahrungen anbieten (Nordrhein-Westfalen 2021). Neben Hochschulen existieren viele weitere staatlich anerkannte Bildungseinrichtungen, die zertifizierte berufliche Weiterbildungen offerieren. Die Zertifizierung von Bildungsträgern erfolgt durch externe private Prüfstellen (fachkundige Stellen bzw. Zertifizierungsstellen). Diese werden durch die Deutsche Akkreditierungsstelle (DAkkS) akkreditiert und überwacht (Deutscher Bildungsserver 2021; Doerr und Kruppe 2012). Derzeit gibt es 34 fachkundige Stellen, wie den TÜV (Nord, Süd, Rheinland) oder die HZA Hanseatische Zertifizierungsagentur (Bundesagentur für Arbeit 2021b). Als Vermittler von Onlinekursen und Zertifikatslehrgängen für Weiterbildungen agiert die Bundesagentur für Arbeit über ihre Kursnet-Datenbank (Bundesagentur für Arbeit 2021a). Darüber hinaus gibt es eine Vielzahl an weiteren Bildungseinrichtungen, die berufliche Weiterbildungen ohne staatliche Zertifizierung anbieten.

Unter Hochschulen und Bildungseinrichtungen, die spezifische Weiterbildungen für Data Science bzw. ML anbieten, sind beispielsweise die Technische Universität Dortmund, die Ludwig-Maximilians-Universität München, die Westfälische WilhelmsUniversität Münster und die Zürcher Hochschule für angewandte Wissenschaften, die TDWI-Akademie, die Bitkom Akademie und die Fraunhofer Academy vertreten. 
Auf der Grundlage der identifizierten aktuellen beruflichen Weiterbildungsmöglichkeiten wird beispielsweise im Rahmen des ML2KMU-Projekts ${ }^{1}$ ein Schulungskatalog aufgebaut. Dabei werden Weiterbildungen berücksichtigt, welche die ausgeprägten Kompetenzen aus Abb. 1 adressieren. Der Katalog vereint das heterogene Feld der aktuellen Weiterbildungsmöglichkeiten in einer Datenbank und erlaubt es, Angebote anhand der individuellen Spezifikationen zu vergleichen. Dadurch können die für den jeweiligen vorliegenden Qualifikationsbedarf am besten geeigneten Weiterbildungen abgeleitet werden. Dazu werden die Kurse zum einen entsprechend ihrer Charakteristiken eingeordnet, wie Dauer, Aufwand, Kosten, Zugangsvoraussetzungen, Zielgruppe, Kursformat und Anwesenheitsform, Sprache, Fachgebiet sowie Abschlusszertifizierung. Bei MOOC-Plattformen wird die Integration anhand der verschiedenen Kursanbieter weiter aufgegliedert. Zum anderen werden die Kurse bezüglich ihres fachlichen Einstiegsniveaus sowie des Niveaus der zu erreichenden Qualifizierung in den einzelnen Kompetenzen bewertet. Die Bewertung wird dabei entsprechend den in Abschn. 2 ausgeprägten Kompetenzen und den vorgestellten Bewertungsstufen von (0) bis (4) vorgenommen, um einen konsistenten Abgleich mit den Kompetenzprofilen der einzelnen Rollen eines MLTeams zu gewährleisten. Auf diese Weise kann das Kursangebot auf das individuelle Anforderungsprofil der Unternehmen und der $\mathrm{zu}$ qualifizierenden Mitarbeiter abgestimmt werden. Auch werden spezielle Weiterbildungsprogramme für Unternehmen berücksichtigt, bei denen einige Anbieter besondere Konditionen gewähren oder individuelle Schulungsprogramme anpassen.

\section{Ableitung zielgerichteter Maßnahmen zur Kompetenzentwicklung für ML-Initiativen}

Das grundlegende Konzept zur Ableitung konkreter Maßnahmen zur Kompetenzentwicklung von Mitarbeitern in ML-Projekten ist in Abb. 2 dargestellt. Die Basis bildet die jeweilige Zielrolle, die ein Mitarbeiter zukünftig in einem ML-Projektteam eines Unternehmen einnehmen soll.

Wie in Abschn. 2 dargelegt wurde, ist jede Rolle mit einem spezifischen Niveau in den einzelnen ausgeprägten Kompetenzen verbunden. Um die jeweilige Rolle demnach hinreichend ausfüllen zu können, muss das spezifische Kompetenzniveau durch den Mitarbeiter mindestens erreicht werden. Es handelt sich dabei also um das Zielkompetenzprofil der Zielrolle. Nachdem die jeweiligen Mitarbeiter den Rollen des ML-Teams zugeordnet worden sind, muss für jeden Mitarbeiter eine Erfassung des Ist-Zustandes der vorhandenen Kompetenzen vorgenommen werden. Dies geschieht mithilfe des in Abschn. 3 vorgestellten Fragebogens. Mit diesem wird eine numerische Bewertung der Mitarbeiter in den ausgeprägten Kompetenzen

1 „Konzept zum Aufbau von Kompetenzen des Maschinellen Lernens für Anlagenhersteller und produzierende $K M U^{\prime \prime}$ gefördert im Rahmen des Doktorandennetzwerks „Digitale Souveränität in der Wirtschaft, Themenbereich Maschinenbau der Zukunft - ein Projekt des Instituts für Innovation und Technik (iit)“. 
anhand der vorgestellten Bewertungsstufen vorgenommen, was durch den Mitarbeiter zusammen mit seinen disziplinarischen und fachlichen Vorgesetzten erfolgen kann. Aufgrund der geeigneten Form des Fragenbogens, bei dem die einzelnen ausgeprägten Kompetenzen tabellarisch mit numerischen Werten erfasst werden, bietet sich die Möglichkeit, den Fragebogen zu einer Qualifizierungsmatrix zu erweitern. Die Qualifizierungsmatrix stellt ein geeignetes Instrument zur Identifikation von Entwicklungszielen dar (bfw - Unternehmen für Bildung 2014). Ein Überblick über die Qualifizierungsmatrix ist beispielhaft für die Rolle eines Citizen Data Scientist als Orchestrierer mit dem Profil eines exemplarischen Mitarbeiters in Abb. 2 dargestellt. Die Qualifizierungsmatrix bietet die Möglichkeit, das erforderliche Kompetenzprofil einer Zielrolle dem erfassten Kompetenzprofil eines Mitarbeiters gegenüberzustellen. Dies erlaubt einen systematischen Soll- und Ist-Vergleich des Kompetenzprofils zwischen den Rollen und den zugewiesenen Mitarbeitern. Aus der numerischen Differenz des Soll- und Ist-Profils können dann die qualitativen und quantitativen Qualifizierungs- und Entwicklungsbedarfe identifiziert werden. Zugleich bietet das die Möglichkeit, Potenziale von Mitarbeitern zu entdecken, sollte das Zielprofil signifikant übertroffen werden. Die einzelnen Kompetenzen, in denen allerdings ein Defizit besteht, müssen vom vorhandenen Niveau auf das Mindestniveau der entsprechenden Rolle weiterentwickelt werden. Dies kann zielgenau mithilfe der Weiterbildungen des Schulungskatalogs erreicht werden. Die Güte der Weiterbildungen für den jeweiligen Anwendungsfall kann daran bemessen werden, welche Weiterbildung die vorhandene Differenz des numerischen Soll-Ist-Vergleichs am meisten behebt. Um Ressourcen zu sparen, könnte darauf geachtet werden, dass möglichst wenig Kompetenzen, die bereits dem Zielniveau genügen, zusätzlich parallel weitergebildet werden. Außerdem können alle Weiterbildungen herausgefiltert werden, bei dem das Einstiegsniveau nicht erfüllt wird. Auch bestünde die Möglichkeit, einzelne Kompetenzen isoliert zu schulen. In jedem Fall werden die Weiterbildungsangebote so vorgefiltert, dass sie den Mitarbeiter zu der entsprechenden Rolle hinreichend qualifizieren.

Damit die geeignetsten Weiterbildungsangebote identifiziert werden können, sind zusätzlich noch die weiteren Kriterien nach Abschn. 4 zu berücksichtigen. Dabei werden Filter integriert, welche die Auswahl nach dem Kursformat mit der entsprechenden Anwesenheitsform eingrenzen. Dabei ist es zum Beispiel wichtig, ob Unternehmensfortbildungen für eine Gruppe oder individuelle Weiterbildungen gewünscht sind. Weitere wichtige Kriterien sind der Zeitraum und die Dauer, der Aufwand, die Kosten und die Kurssprache. Auch die Zugangsvoraussetzungen müssen erfüllt sein und möglicherweise ist eine bestimmte Abschlusszertifizierung erforderlich. Ein weiteres Auswahlkriterium stellt der Kursanbieter selbst dar, sofern ein bestimmter Anbieter favorisiert wird. Die Priorisierung der Auswahl erfolgt also, indem zunächst die Effektivität der Weiterbildungsmaßnahmen und anschließend die Effizienz anhand der individuellen Rahmenbedingungen bewertet wird. Um eine schnelle und einfache Anwendung der Plattform zu ermöglichen, kann der Anwender einen Defaultzustand wählen, bei dem keine Selbsteinschätzung notwendig ist und alle Ist-Kompetenzen mit der Stufe (0) bewertet werden. Dadurch erhält der Mitarbeiter einen schnellen Überblick über alle verfügbaren Weiterbildungsmaßnahmen, welche die jeweiligen Kompetenzen auf das erforderliche Mindestmaß der Rolle qualifizieren. 


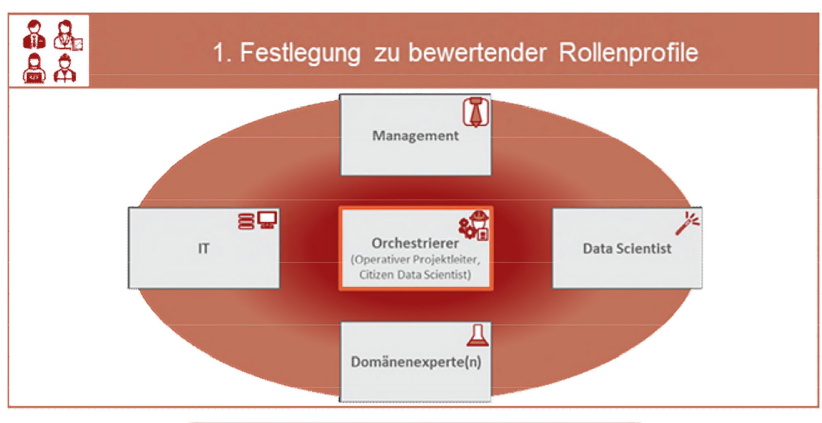

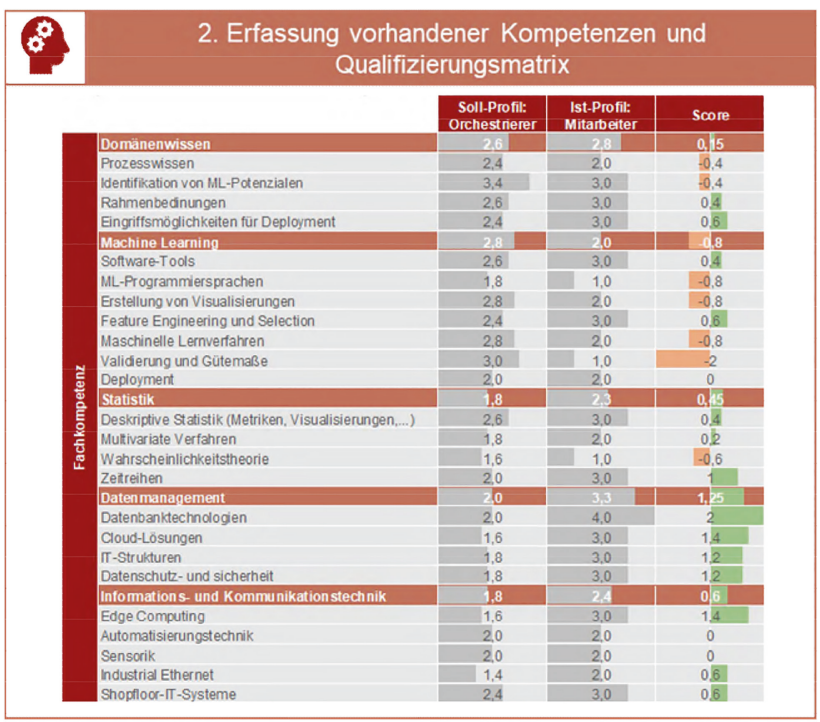

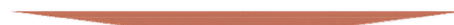

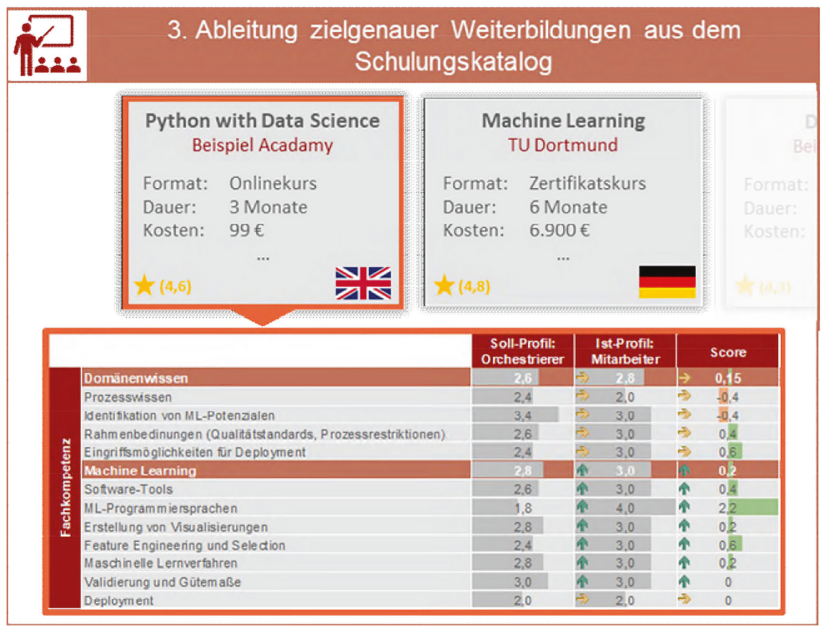

Abb. 2. Konzept zur Ableitung von Maßnahmen zur ML-Kompetenzentwicklung 


\section{Zusammenfassung und Ausblick}

Im Rahmen des Forschungsvorhabens ML2KMU wurde ein Konzept entwickelt, mit dem Unternehmen zielgerichtet Maßnahmen zum Aufbau von Kompetenzen ableiten können, die für ML-Initiativen notwendig sind. Dies erfolgt infolge der zunehmenden Komplexität in der Regel durch interdisziplinäre Teams, die verschiedene Fachbereiche abdecken. Für die generelle Zusammensetzung solcher ML-Teams wurden die einzelnen Rollen definiert und die erforderlichen Kompetenzen ausgeprägt. Dabei wurde zu jeder Rolle ein spezifisches Kompetenzprofil erstellt, das als Zielprofil einen Überblick über das notwendige Mindestniveau in den einzelnen Kompetenzen bietet. Anschließend wurden die verschiedenen Methoden zur Erfassung vorhandener Kompetenzen von Mitarbeitern vorgestellt. Dabei wurde die schriftliche Befragung als optimale Methode für das vorliegende Konzept ausgewählt. Diese wird in Form einer numerischen Bewertung für einen Mitarbeiter in den ausgeprägten einzelnen Kompetenzen durchgeführt. Die Bewertung lässt sich in eine Qualifizierungsmatrix überführen, mit der anschließend ein Soll-Ist-Kompetenzvergleich für die einzelnen Rollen vorgenommen werden kann. Aus der numerischen Differenz zwischen dem Soll- und Ist-Kompetenzniveau lassen sich dann die Kompetenzlücken bei einer Zielrolle identifizieren. Daneben wird ein Schulungskatalog aufgebaut, der die verschiedenen Möglichkeiten an Weiterbildungsangeboten entsprechend ihrer Charakteristiken enthält. Elementar ist hierbei, dass die verschiedenen Weiterbildungsangebote bezüglich des Einstiegs- und des Qualifizierungsniveaus anhand des Bewertungsschemas für die einzelnen Kompetenzen bewertet werden. Dadurch wird es dann möglich, die Kompetenzlücke eines Mitarbeiters bei seiner Zielrolle durch geeignete Weiterbildungen zu adressieren. Gleichzeitig können auch anhand der Kurscharakteristiken Rahmenbedingungen, wie Dauer, Preis, Anwesenheitsform, Qualifizierung oder Kurssprache, berücksichtigt werden. Auch spezielle Angebote für Unternehmen können dabei Beachtung finden.

Als nächstes wird das vorgestellte Konzept in ein Web-basiertes Tool überführt und KMU-gerecht aufbereitet. Dies eröffnet die Möglichkeit, die Kompetenzentwicklungsmaßnahmen aktuell zu halten sowie KMU den Zugang in Form einer Navigationshilfe zu vereinfachen. Dabei wird die Qualifizierungsmatrix als OnlineAssessment integriert. Die Website wird diskriminierungsfrei zur Verfügung gestellt und dient ebenfalls der Information über Projektinhalte und aktuelle Forschungsergebnisse.

Daneben wird eine ML-Grundlagenschulung in Form eines Strategieworkshops konzipiert. Hierzu wird ein Demonstrator als IoT-Testumgebung aufgebaut. Der Demonstrator besteht aus einer cyber-physischen Mikrobrauerei mit industrieller Steuerungstechnik, in der eine datengetriebene Optimierung von Rezepturen mithilfe von ML umgesetzt wird. Zudem soll ein allgemeines Verständnis für komplexe Muster und Zusammenhänge von Ursache und Wirkung im Brauwesen gewonnen werden. Am greifbaren Beispiel von Brauereiprozessen bietet der Demonstrator eine ideale Möglichkeit, um praktische Erfahrungen im Bereich ML zu vermitteln. Damit soll die Relevanz der ausgeprägten ML-Kompetenzen und Rollen demonstriert werden. Daneben soll dies eine Möglichkeit sein, Strategien für zukünftige ML-Ansätze in den eigenen Produkten und Prozessen abzuleiten. Diese Praxiseindrücke sollen als Impulsgeber für zukünftige Geschäftsmodelle und einen vertieften Einsatz von ML-Methoden dienen. 


\section{Literatur}

acatech: Kompetenzentwicklungsstudie Industrie 4.0. Erste Ergebnisse und Schlussfolgerungen. (Hrsg.) v. acatech - DEUTSCHE AKADEMIE DER TECHNIKWISSENSCHAFTEN, Fraunhofer-Institut für Materialfluss und Logistik IML und equeo GmbH. München (2016)

Bauer, N., Stankiewicz, L., Jastrow, M., Horn, D., Teubner, J., Kersting, K. et al.: Industrial data science. Developing a qualification concept for machine learning in industrial production. In: European Conference on Data Analysis (ECDA). Paderborn, 04.-06.07.2018 (2018)

Bertelsmann Stiftung: Zukunft der Arbeit in deutschen KMU (2018)

bfw - Unternehmen für Bildung: Leitfaden für die Erstellung von passgenauen Qualifizierungen. Erstellt im Rahmen des vom BMBF geförderten Projekts Prokom-4.0. Berufsfortbildungswerk Gemeinnützige Bildungseinrichtung des DGB GmbH (bfw). Erkrath (2014)

Bloom, B.S., Engelhart, M.D. (Hrsg.): Taxonomie von Lernzielen im kognitiven Bereich. 35Beltz-Studienbuch, 5. Aufl., Bd. 35. (17. - 21. Tsd.). Beltz, Weinheim (1976)

Boockmann, B., Maier, A., Schafstädt, C.: Vereinbarungen der Sozialpartner zur Weiterbildung - ein Blick in ausgewählte Branchen. In: Bertelsmann Stiftung (Hrsg.) Gütersloh (2021)

Bundesagentur für Arbeit: KURSNET - Beruflich weiterbilden. https://kursnet-finden.arbeitsagentur.de/kurs/portal/bildungssuchende/beruflichWeiterbilden.do (2021a). Zugegriffen: 26. Juli 2021

Bundesagentur für Arbeit: Verzeichnis der fachkundigen Stellen zur Anerkennung von Bildungsträgern und -maßnahmen nach SGB III. https://kursnet-finden.arbeitsagentur.de/ kurs/start?target=fks (2021b). Zugegriffen: 26. Juli 2021

Castellano, S.: MOOCs in the workplace. https://www.td.org/magazines/td-magazine/moocs-inthe-workplace (2014). Zugegriffen: 25. Juli 2021

Czernich, N., Fackler, T., Falck, O., Schüller, S., Wichert, S., Keveloh, K., Vijayakumar, R.M.: Digitale Kompetenzen - Ist die deutsche Industrie bereit für die Zukunft? Ifo Institut. (2019)

Dettmers, S., Jochmann, W., Zimmermann, T., Knappstein, M., Fastenroth, L.M., Heming, J.: Future skills - future learning. Eine Kooperationsstudie von Kienbaum mit StepStone. (Hrsg.) v. StepStone GmbH und Kienbaum Institut @ ISM für Leadership \& Transformation GmbH. https://www.kienbaum.com/de/publikationen/future-skills-future-learning/ (2021). Zugegriffen: 2. Aug. 2021

Deuse, J., Erohin, O., Lieber, D.: Wissensentdeckung in vernetzten, industriellen Datenbeständen. In: Lödding, H. (Hrsg.) Industrie 4.0. Wie intelligente Vernetzung und kognitive Systeme unsere Arbeit verändern. 27. HAB-Forschungsseminar. Hamburg, 12.-13.09.2014 S. 373-395. Gito (Schriftenreihe der Hochschulgruppe für Arbeits- und Betriebsorganisation e. V. (HAB)), Berlin (2014)

Deuse, J., Wöstmann, R., Schulte, L., Panusch, T., Kimberger, J.: Transdisciplinary competence development for role models in data-driven value creation. The citizen data scientist in the centre of industrial data science teams. In: Sinh, W., Schlund, S. (Hrsg.) Kompetenzentwicklung und Lernassistenzsysteme für die datengetriebene Zukunft, 03.-04.09.2021. Wissenschaftliche Gesellschaft für Arbeits- und Betriebsorganisation (WGAB) e. v. GITO Verlag, in Veröffentlichung, Berlin (2021)

Deutscher Bildungsserver: Anerkennung von Bildungsträgern nach SGB III. https://www. bildungsserver.de/Anerkennung-von-Bildungstraegern-nach-SGB-III-5096-de.html (2021). Zugegriffen: 26. Juli 2021

Dodson, M.N., Kitburi, K., Berge, Z.L.: Possibilities for MOOCs in corporate training and development. Perf. Improv. 54(10), 14-21 (2015). doi:https://doi.org/10.1002/pfi.21532 
Doerr, A., Kruppe, T.: Bildungsgutscheine und Zertifizierung aus Sicht der Arbeitsverwaltung. Nürnberg: Institut für Arbeitsmarkt- und Berufsforschung (IAB). IAB-Forschungsbericht, 6/2012. https://www.econstor.eu/handle/10419/84919 (2012)

Döring, N., Bortz, J.: Forschungsmethoden und Evaluation in den Sozial- und Humanwissenschaften. Unter Mitarbeit von Sandra Pöschl-Günther (5. vollständig überarbeitete, aktualisierte und erweiterte Aufl.). Springer (Springer-Lehrbuch), Berlin. https://link. springer.com/content/pdf/10.1007\%2F978-3-642-41089-5.pdf (2016). Zugegriffen: 23. Juli 2021

Edukatico: MOOCs auf Deutsch: Welche Kurse gibt es? https://www.edukatico.org/de/report/ moocs-auf-deutsch-welche-kurse-gibt-es (2021). Zugegriffen: 24. Juli 2021

Eickelmann, M., Wiegand, M., Konrad, B., Deuse, J.: Die Bedeutung von Data Mining im Kontext Industrie 4.0. Z. wirtschaftlichen Fabrikbetrieb (ZWF) 110(11), 738-743 (2015)

Fayyad, U., Piatetsky-Shapiro, G., Smyth, P.: From data mining to knowledge discovery in databases. AIMag 17(3), 37 (1996). doi:https://doi.org/10.1609/aimag.v17i3.1230

Gillmann, B.: Digitale Arbeitswelt: Handelsblatt Research Institut: „Bei der Weiterbildung versagt der Markt“. In: Handelsblatt, 16.04.2021. https://www.handelsblatt.com/politik/deutschland/ handelsblatt-research-institut-hri-studie-staat-muss-den-voellig-unuebersichtlichen-marktfuer-weiterbildungklarer-strukturieren/27094450.html?nlayer=Themen_11804704 (2021). Zugegriffen: 3. Aug. 2021

Kaplan, A.M., Haenlein, M.: Higher education and the digital revolution: about MOOCs, SPOCs, social media, and the cookie monster. Bus. Horiz. 59(4), 441-450 (2016). doi:https://doi.org/10.1016/j.bushor.2016.03.008

Leopold, T.A., Zahidi, S., Ratcheva, V.S.: The future of jobs report 2018. In: World Economic Forum (Hrsg.). Cologny/Genf, Schweiz (2018)

Lewin, T.: Universities Abroad Join MOOC Course Projects. In: The New York Times, 2013. https://www.nytimes.com/2013/02/21/education/universities-abroad-join-mooc-courseprojects.html (2013). Zugegriffen: 25. Juli 2021

McAuley, A., Stewart, B., Siemens, G., Cormier, D.: Massive Open Online Courses. Digital ways of knowing and learning. The MOOC model for digital practice. In: University of Prince Edward Island (Hrsg.). Charlottetown, Kanada (2010)

Morik, K., Deuse, J., Stolpe, M., Bohnen, F., Reichelt, U.: Einsatz von Data-Mining-Verfahren im Walzwerk. stahl und eisen 130(10), 80-82 (2010)

Nielson, B.: The year of the corporate MOOC? (Hrsg.) v. LinkedIn. https://www.linkedin. com/pulse/20140320165303-16131081-2014-the-year-of-the-corporate-mooc (2014). Zugegriffen: 25. Juli 2021

Nöhring, F.: Ansatz zur zielgerichteten Gestaltung cyber-physischer Produktionssysteme für kleine und mittlere Unternehmen. Shaker (2021)

Nordrhein-Westfalen: § 62 HG - Wissenschaftliche und künstlerische Weiterbildung. In: Gesetz über die Hochschulen Landes Nordrhein-Westfalen (Hochschulgesetz - HG). http://www. lexsoft.de/cgi-bin/lexsoft/justizportal_nrw.cgi?xid=2566366,63 (2021). Zugegriffen: 26. Juli 2021

North, K., Brandner, A., Steininger, T.: Die Wissenstreppe: Information - Wissen - Kompetenz. In: North, K., Brandner, A., Steininger, T. (Hrsg.) Wissensmanagement für Qualitätsmanager S. 5-8. Springer Fachmedien Wiesbaden (essentials), Wiesbaden (2016)

Obmann, C.: Förderung Fortbildung: Die wichtigsten Weiterbildungs-Zuschüsse. In: Handelsblatt, 10.07.2021. https://www.handelsblatt.com/karriere/digitale-transformation-diesegefoerderten-weiterbildungsprogramme-ebnen-den-weg-in-die-digitale-welt/27403808. html?ticket=ST-12609193-TaE2Qf1DFcxH5EntgbTO-ap4 (2021). Zugegriffen: 3. Aug. 2021

Pappano, L.: Massive open online courses are multiplying at a rapid pace. In: The New York Times, 2012. https://www.nytimes.com/2012/11/04/education/edlife/massive-open-onlinecourses-are-multiplying-at-a-rapid-pace.html (2012). Zugegriffen: 23. Juli 2021 
Plattform Lernende Systeme: KI im Mittelstand. Potenziale erkennen, Voraussetzungen schaffen, Transformation meistern. (Hrsg.) v. Lernende Systeme - Die Plattform für Künstliche Intelligenz. https://www.plattform-lernende-systeme.de/files/Downloads/Publikationen/PLS_ Booklet_KMU.pdf (2021). Zugegriffen: 13. Aug. 2021

RapidMiner: Building the perfect AI team. https://rapidminer.com/resource/building-ai-team/ (2020)

Reckelkamm, T., Deuse, J.: Kompetenzentwicklung für Maschinelles Lernen zur Konstituierung der digitalen Souveränität. In: Hartmann, E.A. (Hrsg.) Digitalisierung souverän gestalten. Innovative Impulse im Maschinenbau S. 31-43. Springer Vieweg, Berlin (2021)

Saltz, J.S., Grady, N.W.: The ambiguity of data science team roles and the need for a data science workforce framework. In: 2017 IEEE International Conference on Big Data (Big Data) S. 2355-2361. IEEE, Boston 11.12.2017-14.12.2017 (2017)

Schulte, L., Schmitt, J., Stankiewicz, L., Deuse, J.: Industrial data science. Interdisciplinary competence for machine learning in industrial production. In: Schüppstuhl, T., Tracht, K., Henrich, D. (Hrsg.) Annals of scientific society for assembly, handling and industrial robotics S. 161-171. Bayreuth, 11-12.03.2020. Springer Vieweg, Berlin (2020)

Siemens, G.: Massive open online courses: innovation in education? In: McGreal, R., Kinuthia, W., Marshall, S. (Hrsg.) Commonwealth of learning, Athabasca University, S. 5-16. https://oerknowledgecloud.org/sites/oerknowledgecloud.org/files/pub_PS_OER-IRP_web. pdf\#page $=31(2013)$

Stark, R., Deuse, J., Damerau, T., Reckelkamm, T., Lindow, K.: Data preparation for data analytics (DPDA). Arbeitsgruppe des prostep ivip e. V. News. Wiss. Ges. Produktentwicklung 2, 4-6. http://www.wigep.de/fileadmin/download/wigep/WiGeP-News_2_2019_01.pdf (2019)

Zahidi, S., Ratcheva, V.S., Hingel, H., Brown, S.: The future of jobs report 2020. In: World Economic Forum (Hrsg.). Cologny/Genf, Schweiz (2020)

Zschech, P., Fleißner, V., Baumgärtel, N., Hilbert, A.: Data science skills and enabling enterprise systems. HMD 55(1), 163-181 (2018). doi:https://doi.org/10.1365/s40702-0170376-4

Open Access Dieses Kapitel wird unter der Creative Commons Namensnennung 4.0 International Lizenz (http://creativecommons.org/licenses/by/4.0/deed.de) veröffentlicht, welche die Nutzung, Vervielfältigung, Bearbeitung, Verbreitung und Wiedergabe in jeglichem Medium und Format erlaubt, sofern Sie den/die ursprünglichen Autor(en) und die Quelle ordnungsgemäß nennen, einen Link zur Creative Commons Lizenz beifügen und angeben, ob Änderungen vorgenommen wurden.

Die in diesem Kapitel enthaltenen Bilder und sonstiges Drittmaterial unterliegen ebenfalls der genannten Creative Commons Lizenz, sofern sich aus der Abbildungslegende nichts anderes ergibt. Sofern das betreffende Material nicht unter der genannten Creative Commons Lizenz steht und die betreffende Handlung nicht nach gesetzlichen Vorschriften erlaubt ist, ist für die oben aufgeführten Weiterverwendungen des Materials die Einwilligung des jeweiligen Rechteinhabers einzuholen.

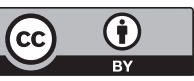

